International Journal of Design and Manufacturing

Technology (IJDMT),

ISSN 0976 - 6995(Print), ISSN 0976 - 7002(Online)

Volume 2, Issue 1, January-December (2011), pp. 01-06

(C) IAEME, http://www.iaeme.com/ijdmt.html

\title{
SVM BASED FAULT DIAGNOSIS OF MONOBLOCK CENTRIFUGAL PUMP USING STATIONARY WAVELET FEATURES
}

\author{
V. Muralidharan ${ }^{1,2}$, V.Sugumaran ${ }^{1}$, Gaurav Pandey ${ }^{1}$ \\ ${ }^{1}$ Department of Mechatronics, SRM University, Kattankulathur, 603203. \\ ${ }^{2}$ Research Scholar, Karpagam University, Coimbatore
}

\begin{abstract}
Fault diagnosis of monoblock centrifugal pump essentially forms a pattern recognition problem. There are three important steps to be performed in pattern recognition namely feature extraction, feature selection and classification. In this study, stationary wavelet transform (SWT) is used for feature extraction and SMO algorithm (a WEKA implementation of Support Vector Machine (SVM) algorithm) is used for classification. The different fault conditions considered for the present study are cavitation (CAV), impeller fault (FI), bearing fault (BF) and both impeller and bearing fault (FBI). The representative signal is acquired for all faulty conditions, features are extracted, classified and the results are presented. The experimental set up and the procedure for conducting the experiments are discussed in detail.
\end{abstract}

Keywords: Stationary wavelets transform, fault diagnosis, wavelet feature, SMO algorithm.

\section{INTRODUCTION}

In a monoblock centrifugal pump, defective bearing, defect on the impeller and cavitation cause a very serious problems. Cavitation results in undesirable effects, such as deterioration of hydraulic performance (drop in head capacity and efficiency). Fault detection is achieved by comparing the signals of monoblock centrifugal pump running under normal and faulty conditions. Vibration signals are widely used in condition monitoring of centrifugal pumps. For the measurement of the vibration levels for each condition, seismic or piezoelectric transducers along with data acquisition system is used to capture the vibration signals. From the vibration signal relevant features are extracted using Stationary Wavelet Transformations (SWT) and classification is done using SMO algorithm and the results are presented.

Jiangping Wang, Hongtao Hu (2006) focuses on a problem of vibration-based condition monitoring and fault diagnosis of pumps. The vibration-based machine condition monitoring and fault diagnosis incorporate a number of machinery fault detections and diagnostic techniques. They used fuzzy logic principle as a fault diagnostic technique to describe the uncertain and ambiguous relationship between different fault symptoms and classify frequency spectra representing various pump faults. Fansen Kong, Ruheng Chen (2004) proposed a new combined method based on wavelet transformation, fuzzy logic and 
International Journal of Design and Manufacturing Technology (IJDMT), ISSN 0976 - 6995(Print), ISSN 0976 - 7002(Online) Volume 2, Issue 1, January-December (2011), (C IAEME

neuro-networks for fault diagnosis of a triplex pump. The failure characteristics of the fluidand dynamic-end can be divided into wavelet transform in different scales. Therefore, the characteristic variables can be constructed making use of the coefficients of Edgeworth asymptotic spectrum expansion formula and fuzzified to train the neuro-network to identify the faults of fluid- and dynamic-end of triplex pump in fuzzy domain. Tests indicate that the information of wavelet transformation in scale 2 is related to the meshing state of the gear and the information in scales 4 and 5 is related to the running state of fluid-end. Good agreement between analytical and experimental results has been obtained. N.R. Sakthivel, V. Sugumaran, Binoy. B. Nair (2010) presented the use of decision tree and rough sets to generate the rules from statistical features extracted from vibration signals under good and faulty conditions of a mono-block centrifugal pump. A fuzzy classifier was built using decision tree and rough set rules and tested using test data. The results obtained using decision tree rules and those obtained using rough set rules were compared. Finally, the accuracy of a principle component analysis based decision tree-fuzzy system was also evaluated. The study revealed that overall classification accuracy obtained by the decision tree-fuzzy hybrid system was to some extent better than the rough set-fuzzy hybrid system.

\section{EXPERIMENTAL STUDIES}

The main idea of this study is to find whether the monoblock centrifugal pump is in good condition or in faulty condition by a systematic procedure following certain steps. If the pump is found to be in faulty condition then the next step is to segregate the faults into cavitation, bearing fault, impeller defect, bearing and impeller defect together.

\subsection{EXPERIMENTAL SETUP DESCRIPTION}

The monoblock centrifugal pump is taken for this study. The motor (2HP) is used to drive the pump. Piezoelectric type accelerometer is used to measure the vibration signals. The accelerometer is mounted on the pump inlet using adhesive and connected to the signal conditioning unit where signal goes through the charge amplifier and an analog to digital converter (ADC) and the signal is stored in the memory. Then the signal is processed from the memory and it is used to extract the features.

\subsection{PROCEDURE}

The pump was allowed to rotate at a speed of $2880 \mathrm{rpm}$ at normal working condition and the vibration signals are measured. The sampling frequency of $24 \mathrm{KHz}$ and sample length of 1024 were considered for all conditions of pump. The sample length was chosen arbitrarily to an extent; however, the following points were considered. After calculating the wavelet transforms it would be more meaningful when the number of sample is more .On the other hand, as the number of sample is increases, the computation time increases .To strike a balance, sample length of around 1,000 was chosen. The specification of the monoblock centrifugal pump is given as below. 
International Journal of Design and Manufacturing Technology (IJDMT), ISSN 0976 - 6995(Print), ISSN 0976 - 7002(Online) Volume 2, Issue 1, January-December (2011), (C IAEME

Table 1 Specification of the pump under study

\begin{tabular}{|l|l|l|l|}
\hline Rotational Speed & 2880 r.p.m & Pump Size & $\begin{array}{l}50 \mathrm{~mm} \text { x 50 } \\
\mathrm{mm}\end{array}$ \\
\hline Current & $11.5 \mathrm{~A}$ & Discharge & $392 \mathrm{lps}$ \\
\hline Head & $20 \mathrm{~m}$ & Power & $2 \mathrm{hp}$ \\
\hline
\end{tabular}

In the present study the following faults were simulated as described below.

Cavitation - by intentionally closing the suction gate valve partially.

Impeller fault - chipping to dislodge one material to simulate pitting.

bearing fault - a thin cut through wire cut EDM.

Bearing and Impeller fault together.

The faults were introduced one at a time and vibration signals were taken.

\section{FEATURE EXTRACTION}

Stationary Wavelet Transform (SWT) has been widely used and provides the physical characteristics of time-frequency domain data. The advantage of using SWT is that it avoids the down sampling process and hence one can preserve the length of the signal for analysis. SWT of different versions of different wavelet families have been considered. The following wavelet families and their sub families have been tried for the present study. Daubechies wavelet, Coiflet, biorthogonal wavelet, reversed biorthogonal wavelet, symlets and meyer wavelet.

\section{FEATURE DEFINITION}

Feature extraction constitutes computation of specific measures, which characterize the signal. The stationary wavelet transform (SWT) provides an effective method for generating features. The collection of all such features forms the feature vector. A feature vector is given by

$$
v^{S W T}=\left\{v_{1}^{s w t}, v_{2}^{s w t}, v_{3}^{s w t} \ldots v_{10}^{s w t}\right\}^{T}
$$

A component in the feature vector is related to the individual resolutions by the following equation

$$
v^{s w t}=\frac{1}{n_{i}} w^{2} \quad, i=1,2 \ldots 10
$$

where, ${ }_{i}^{\text {swt }}$ is the ith feature element in a SWT feature vector. $n_{\mathrm{i}}$ is the number of $v$

samples in an $\mathrm{w}^{2}$ individual sub-band.

\section{CLASSIFICATION}

The SVM is a new generation learning system based on statistical learning theory. SVM belongs to the class of supervised learning algorithms in which the learning machine is given a set of features (or inputs) with the associated labels (or output values). Each of these features can be looked upon as a dimension of a hyper- plane. SVMs construct a hyper-plane that separates the hyper-space into two classes (this can be extended to multiclass problems). While doing so, SVM algorithm tries 
International Journal of Design and Manufacturing Technology (IJDMT), ISSN 0976 - 6995(Print), ISSN 0976 - 7002(Online) Volume 2, Issue 1, January-December (2011), (C IAEME

to achieve maximum separation between the classes. Separating the classes with a large margin minimizes the expected generalization error. Intuitively, such a classifier is one, which achieves maximum separation-margin between the classes. SVM 'learning', means that finding a hyper-plane, which maximizes the margin and minimizes the misclassification error. (For detailed description refer [5]).

\section{RESULTS AND DISCUSSION}

All the wavelet families and its sub-groups were used to find the stationary wavelet transform which form the feature vectors. The extracted features were then given as an input to the classifier (SMO algorithm) and the classification accuracies were found. Fig. 1 will describe the classification accuracies among the different families of wavelet. One can easily understand that the classification accuracy of reverse bi-orthogonal family is high. The performance among the reverse bi- orthogonal wavelet and its versions is presented in Fig.2. Form Fig. 2, one can easily understand the maximum classification accuracy is 93.84 which is against rbio3.1. This means that the 3.1 version of reverse biorthogonal wavelet performs relatively better than any other versions of the wavelet families. The detailed classification detail for rbio 3.1 is given below in the form of confusion matrix in Fig. 3.

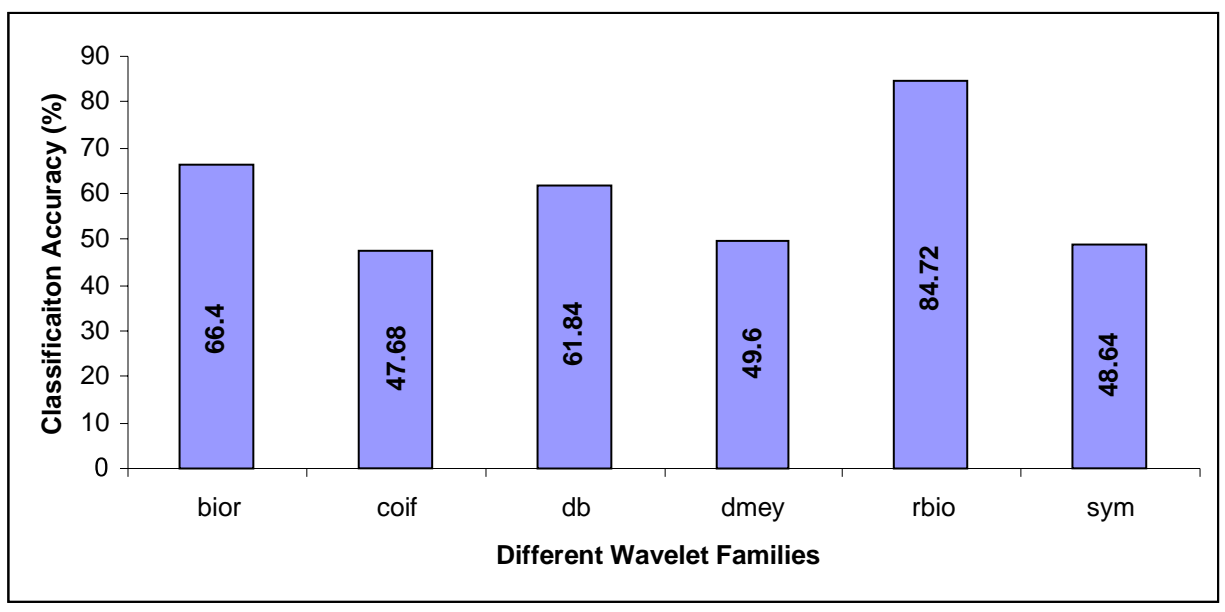

Fig.1. Histogram for classification accuracies for different wavelet families 


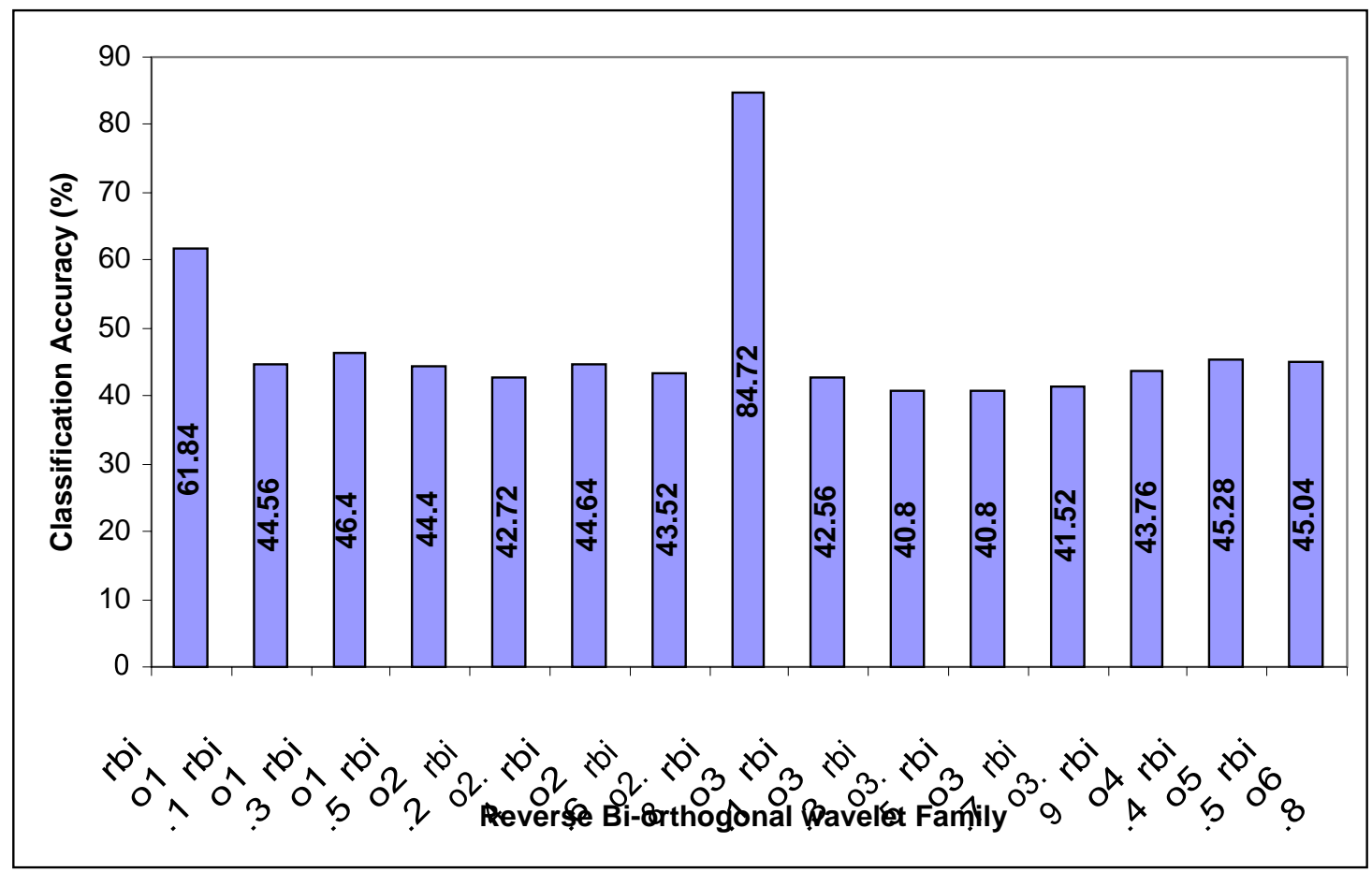

Fig.2. Histogram chart for classification accuracies of rbior wavelet and its versions

\begin{tabular}{ccccc}
\hline $\mathrm{A}$ & $\mathrm{B}$ & $\mathrm{C}$ & $\mathrm{D}$ & $\mathrm{E}$ \\
\hline 245 & 3 & 0 & 2 & 0 \\
6 & 238 & 0 & 6 & 0 \\
0 & 0 & 248 & 0 & 2 \\
167 & 5 & 0 & 78 & 0 \\
0 & 0 & 0 & 0 & 250
\end{tabular}

Fig.3. Confusion matrix for rbio3.1

A - Good; B -Cavitation; C - Impeller Fault (FI); D - Bearing Fault (FB); E - Bearing and Impeller Fault (FBI).

The confusion matrix can be interpreted as follows. The diagonal elements in the matrix are correctly classified and non-diagonal elements are incorrectly classified data points. From the matrix it can be easily understood how it was misclassified and the classification accuracy was calculated accordingly. Therefore, for rbio3.1, the classification accuracy was found to be $84.72 \%$.

\section{CONCLUSION}

In the present study monoblock centrifugal pump was taken for the study. Stationary wavelet features were extracted and classified using SMO algorithm to study the fault discriminating capabilities of wavelets for vibration signal. From the results and discussion one can confidently say that the best wavelet for this application is reversed bi-orthogonal wavelet 3.1 and whose classification accuracy is found to be 84.72. Therefore, to conclude, the SWT features with SMO algorithm based classification will be very much suited for real time applications. 
International Journal of Design and Manufacturing Technology (IJDMT), ISSN 0976 - 6995(Print), ISSN 0976 - 7002(Online) Volume 2, Issue 1, January-December (2011), (C IAEME

\section{REFERENCES}

[1] S. Prabhakar, A. R. Mohanty, A. S Sekhar (2002), "Application of discrete wavelet transforms for detection of ball bearing race faults" Tribology A International,35, 793800.

[2] Jiangping Wang, Hongtao Hu,(2006) “Vibration based fault diagnosis of pump using fuzzy technique "Measurement,39,176-185.

[3] Fansen Kong, Ruheng Chen(2004),"A combined method for triplex pump fault diagnosis based on wavelet transforms ,fuzzy logic and neuro-networks" Mechanical system and signal processing, 18,161-168.

[4] N.R. Sakthivel, V. Sugumaran, Binoy. B. Nair (2010),’Comparison of decision tree fuzzy and rough set fuzzy method for fault categorization of monoblock centrifugal pump", Mechanical system and signal processing,24,1887-1906.

[5] V. Sugumaran, V. Muralidharan, K.I. Ramachandran (2007), "Feature selection using Decision Tree and classification through Proximal Support Vector Machine for fault diagnostics of roller bearing", Mechanical Systems and Signal Processing, 21, 2, 930942.

[6] M. Saimurugan, K.I. Ramachandran, V. Sugumaran, N.R. Sakthivel (2011), "Multi component fault diagnosis of rotational mechanical system based on decision tree and support vector machine " Expert Systems with Applications, 38, 4, 3819- 3826.

[7]V.Sugumaran, K.I. Ramachandran,(2011),"Effects of number of features of classification of roller bearing fault using SVM and PSVM",Experts systems with applications, 38,4, 4088-4096.

[8] D. Zogg, E. Shafai, H.P. Geering(2006),'Fault diagnosis of heat pumps with parameter identification and clustering", Control Engineering practice , 12, 1435-1444. 